\title{
Uveíte das espondiloartropatias: prevalência e relação com doença articular
}

\author{
Uveitis and spondyloarthritis: prevalence and relationship with joint disease
}

\author{
Thelma Larocca Skare ${ }^{1}$ \\ Thiago Quinaglia Silva ${ }^{2}$ \\ Paulo Cesar Pastro ${ }^{3}$
}

\begin{tabular}{|l|}
\hline RESUMO \\
\hline Objetivos: Estudar a prevalência de uveítes na população local de espon- \\
diloartropatias e sua relação temporal com achados articulares. Métodos: \\
Foram revisados prontuários de 77 pacientes com espondiloartropatias \\
atendidos no ambulatório de reumatologia do Hospital Universitário \\
Evangélico de Curitiba, para diagnóstico do tipo de espondiloartropatia, \\
sexo, idade, presença e tipo de uveite, tempo decorrido entre as primeiras \\
manifestações oculares e as articulares. Resultados: Uveíte foi encontra- \\
da em 12 dos 77 pacientes ( $15,6 \%$ ), sendo anterior em $83,3 \%$ dos casos. \\
O aparecimento da uveíte foi semelhante em todas as formas de espon- \\
diloartropatia (p=0,27) e não sofreu influência do sexo do paciente \\
(p=0,74). O tempo médio de aparecimento dos sintomas oculares em \\
relação ao diagnóstico articular foi de 4,04 meses para artrite reativa e 73 \\
meses para espondilite anquilosante (p=0,009). Conclusões: A uveíte \\
encontrada em pacientes com espondiloartropatia é, na sua grande \\
maioria, anterior. O aparecimento da uveíte em relação aos sintomas \\
articulares é mais precoce em casos de artrite reativa do que em espondilite \\
anquilosante.
\end{tabular}

Descritores: Uveíte/etiologia; Manifestações oculares; Espondiloartropatias; Espondilite anquilosante; Artrite reativa

\section{INTRODUC̣̃̃O}

As espondiloartropatias soronegativas (ES) formam um conjunto de doenças reumáticas com características clínicas e laboratoriais comuns. Fazem parte deste grupo: espondilite anquilosante, artrite reativa (ou síndrome de Reiter), artrite psoriática e espondiloartropatias das doenças inflamatórias intestinais (como doença de Crohn e retocolite ulcerativa) $)^{(1)}$. Dentre as características comuns a este grupo, estão as manifestações oculares que podem aparecer como uveítes e conjuntivites ${ }^{(1)}$.

Doenças reumáticas como a artrite reumatóide juvenil, síndrome de Behçet e as ES causam tipicamente uveítes anteriores e respondem por cerca da metade dos casos de uveítes não infecciosas ${ }^{(2)}$. No caso das ES, elas costumam ser unilaterais, não-granulomatosas, agudas e recorrentes ${ }^{(3)}$. Todavia, em ES associadas à artrite psoriática, as uveítes podem ser posteriores, mais prolongadas e bilaterais ${ }^{(4)}$.

As uveítes das ES são eventos imunológicos promovidos por ação das células $\mathrm{T}$ do tipo Th- ${ }^{(5)}$. O elemento inicial que desencadeia o processo é desconhecido embora vários antígenos microbianos e genéticos tenham sido estudados neste contexto. O que está bem estabelecido, é a presença do antígeno de histocompatibilidade classe 1, HLA-B27, o qual está associada 
ao seu aparecimento tanto em populações em que este antígeno de histocompatibilidade é comum ${ }^{(6)}$, como naqueles em que ele é raro ${ }^{(7)}$. Além disto, diferentes subtipos do HLA-B27 apresentam diferentes graus de associação com ocorrência de uveíte $^{(8)}$. Um estudo finlandês mostrou a presença de HLAB27 em até $71 \%$ dos pacientes com uveíte anterior aguda ${ }^{(6)}$. Em estudos realizados em pacientes da Nova Zelândia, foi encontrado esta prevalência em $26 \%$ dos pacientes com uveíte anterior aguda ${ }^{(9)}$. Já no Brasil, um estudo feito em Minas Gerais e englobando 100 pacientes, mostrou uma prevalência de $26 \%$ de HL-B27 positivo em casos de uveíte anterior aguda, dos quais 38\% tinham ES na forma indiferenciada ${ }^{(10)}$.

Por outro lado, a prevalência de uveíte nas diferentes formas de ES varia de acordo com a amostra estudada, o que tem sido atribuído à carga genética da população e, eventualmente, de agentes microbianos, que podem funcionar como gatilho do processo ${ }^{(6,9)}$.

Nesta pesquisa procurou-se verificar a prevalência de uveítes nas diferentes formas de ES em pacientes da cidade de Curitiba, assim como a sua relação temporal com início da doença articular.

\section{MÉTODOS}

O presente estudo foi autorizado pelo comitê de ética em pesquisa da Faculdade Evangélica de Medicina do Paraná protocolado sob número 1.519/2006.

Foram revisados os prontuários de 77 pacientes com ES atendidos no ambulatório de reumatologia do Hospital Universitário Evangélico de Curitiba, no período de janeiro de 2000 a dezembro de 2005. Estes pacientes eram oriundos do Serviço de Atenção Básica à Saúde (75 pacientes) e do Serviço de Oftalmologia do Hospital Universitário Evangélico de Curitiba (2 pacientes).

Todos os pacientes incluídos preenchiam os critérios de classificação para ES propostos pelo European Spondyloarthropathy Study Group (ESSG) ${ }^{(11)}$. Prontuários com dados insuficientes ou preenchidos de maneira incompleta foram excluídos.

Obtiveram-se dados quanto ao tipo de espondiloartropatia diagnosticada, idade de diagnóstico, sexo do paciente, ocorrência de uveíte, localização da uveíte e tempo de ocorrência do primeiro episódio de uveíte em relação ao início dos sintomas articulares.

Os dados foram estudados por tabelas de freqüência e contingência sendo aplicados os testes de Mann-Whitney e Quiquadrado com auxílio do software Graph Pad Prism 4.0. Nível de significância adotado de 5\%.

\section{RESULTADOS}

Dos 77 pacientes estudados, 26 eram mulheres e 51 eram homens com idade média de $42,7 \pm 12,8$ anos. A amostra compunha-se de 1 paciente com espondilite associada à doen- ça inflamatória intestinal; 14 pacientes com espondiloartropatias associadas à artrite psoriática, 17 com artrite reativa e 45 com espondilite anquilosante.

Nestes pacientes foram encontrados 12 casos de uveítes (15,5\%), distribuídos da seguinte maneira: 3 em pacientes com artrite reativa $(17,6 \%$ de todos os pacientes com artrite reativa) e 9 em pacientes com espondilite anquilosante (ou 20\% de todos os pacientes com espondilite anquilosante). Nenhum dos casos de espondiloartropatia associada à psoríase ou doença inflamatória intestinal tinha tido uveíte. Não se encontrou diferença estatística significante entre a prevalência de uveítes nas diferentes formas de espondiloartropatias $(p=0,27)$ (Figura 1).

Todos os casos de uveíte eram de uveítes anteriores com exceção de um paciente com panuveíte e de outro com uveíte intermediária. Estes dois últimos eram pacientes com espondilite anquilosante.

Em relação ao tempo de diagnóstico do primeiro episódio de uveíte e os sintomas articulares notou-se que: em 1 caso, a uveíte apareceu antes dos sintomas articulares; em 1 caso foi concomitante e, em outros 8 , apareceu num período entre 7 a 180 meses após os sintomas articulares (média de 66,7 meses).

Ao se estudar a relação temporal entre uveítes de aparecimento posterior ao diagnóstico da espondiloartropatia encontrou-se que, no caso da espondilite anquilosante este aparecimento foi entre 12 e 180 meses (média de $73 \pm 61,9$ meses) e no caso de artrite reativa entre 0 e 7 meses (média de 4,04 $\pm 2,3$ meses) com $\mathrm{p}=0,009$ (Figura 2).

A distribuição de uveíte de acordo com o sexo do paciente foi de 8 casos nos homens $(15,7 \%)$ e 3 nas mulheres $(11,5 \%)$ com $\mathrm{p}=0,74$.

\section{DISCUSSÃO}

A análise dos presentes resultados mostra uma prevalência de uveíte em 15,6\% em todos os casos de espondiloartropatias sendo em sua grande maioria $(83,3 \%)$ uveíte anterior.

Na tabela 1 encontram-se alguns dados da literatura que

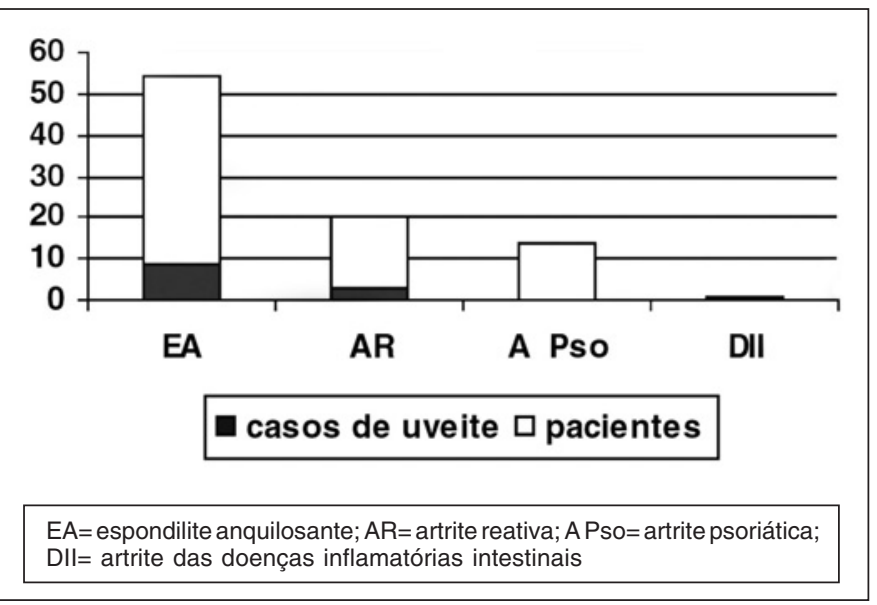

Figura 1 - Uveites nas diferentes formas de espondiloartropatias 


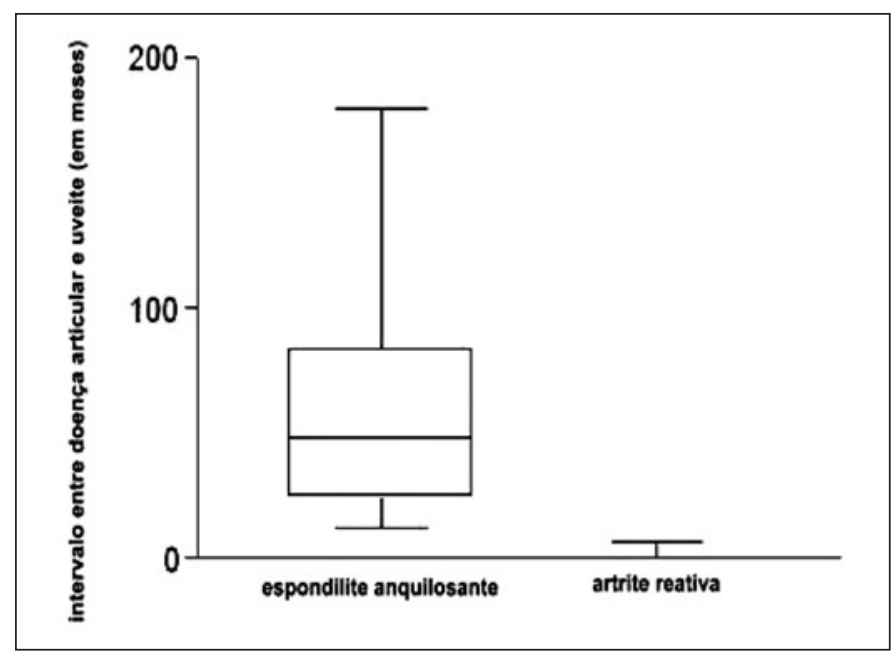

Figura 2 - Relação temporal entre aparecimento de queixas articulares e de uveíte na espondilite anquilosante e na artrite reativa $(p=0,009)$

se referem à chance de uveítes em pacientes com diagnóstico já estabelecido de espondiloartropatias.

Os dados obtidos no presente estudo mostram uma prevalência de $20 \%$ nos casos de espondilite anquilosante e de $17,6 \%$ para artrite reativa, que é menor do que a observada acima (de até 30\% para espondilite anquilosante e de até 37\% para a artrite reativa). Tal fato talvez possa ser explicado em termos da forte ligação deste envolvimento ocular com o HLA-B27, que varia conforme o perfil étnico da população analisada. A prevalência de uveíte anterior em pacientes brasileiros com espondilite anquilosante demonstrou uma taxa bastante parecida com a obtida no presente estudo, em torno de $14,3 \%{ }^{(12)}$.

As uveítes das ES aparecem mais em indivíduos do sexo masculino, porque os estrógenos parecem desempenhar um papel protetor, papel este definido por indução da sintetase constitutiva do óxido nítrico, diminuição na expressão de moléculas de adesão (seletina E) e modulação nos genes que promovem o aparecimento de citocinas pró-inflamatórias como interleucina (IL)-1, IL-6 e fator de necrose tumoral (TNF)alfa ${ }^{(13)}$. Tal fato não pode ser constatado em nossa população, onde a diferença de prevalência não foi significativa entre os sexos. Talvez isso tenha ocorrido pela pequena amostra populacional analisada.

Inflamação articular e ocular não aparecem sempre de

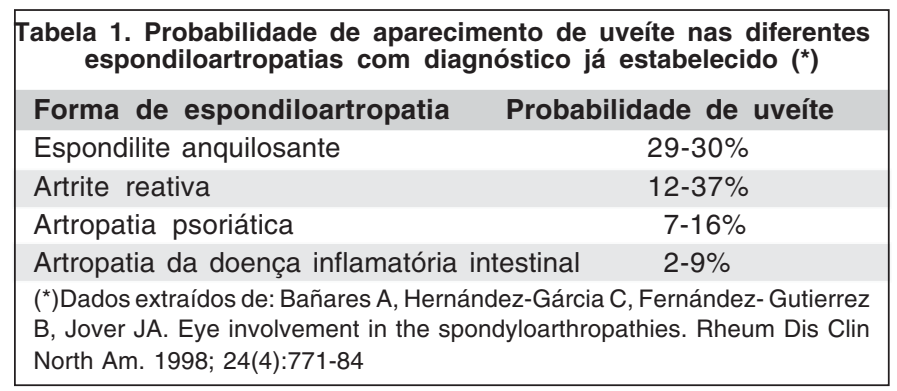

maneira sincrônica e a uveíte pode anteceder, aparecer simultaneamente ou depois das manifestações reumáticas. Pode, inclusive, ser a única manifestação da doença num indivíduo que não tem e nunca terá doença $\operatorname{articular}^{(14)}$. O mais comum, de acordo com a literatura, é que ela apareça de 7 a 10 anos após o início das manifestações músculo-esqueléticas ${ }^{(1)}$. Este dado está de acordo com o observado em nossos pacientes de espondilite anquilosante. Todavia, os pacientes com artrite reativa demonstraram que esta manifestação pode ser muito mais precoce.

\section{CONCLUSÕES}

1) A prevalência de uveíte na população de ES local é menor do que a verificada na literatura mundial.

2) A uveíte da artrite reativa aparece de maneira mais precoce que a uveíte da EA.

3) Embora exista um número maior de pacientes masculinos com espondiloartropatia, o sexo não influi na prevalência de uveíte em nosso meio.

\section{ABSTRACT}

Purpose: To study uveitis prevalence in the local population with spondyloarthritis and its temporal relationship with joint complaints. Methods: We reviewed seventy-seven charts of spondyloarthropathy patients from the rheumatology clinic of the "Hospital Universitário Evangélico de Curitiba" for spondyloarthritis class, patients' sex and age, occurrence of uveitis and its location and relationship between the first episode of uveitis and initial joint complaints. Results: Uveitis was found in 12 of 77 patients (15.6\%) which was anterior in $83.3 \%$ of the cases, without preference for spondyloarthropathy class $(p=0.72)$ and patients' sex $(p=0.74)$. In patients with reactive arthritis, the mean time between uveitis appearance and joint complaints was 4.04 months and in ankylosing spondylitis 73 months $(p=0.009)$. Conclusion: Spondyloarthropathy patients have uveitis that is anterior in most of the cases and that appears earlier in reactive arthritis than in ankylosing spondylitis.

Keywords: Uveitis/etiology; Eye manifestations; Spondylarthropathies; Spondylitis, ankylosing; Arthritis, reactive

\section{REFERÊNCIAS}

1. Bañares A, Hernández-Gárcia C, Fernández-Gutiérrez B, Jover JA. Eye involvement in the spondyloarthropathies. Rheum Dis Clin North Am. 1998;24(4): 771-84.

2. Dick AD. Immune mechanisms of uveitis: insights into disease pathogenesis and treatment. Int Ophthalmol Clin. 2000;40(2):1-18.

3. Bañares A, Jover JA, Fernández-Gutiérrez B, Benítez del Castillo JM, García J, Vargas E, Hernández-García C. Patterns of uveitis as a guide in making rheumatologic and immunologic diagnoses. Arthritis Rheum. 1997;40(2):358-70. 
4. Durrani K, Foster CS. Psoriatic uveitis: a distinct clinical entity? Am J Ophthalmol. 2005;139(1):106-11.

5. Foxman EF, Zhang M, Hurst SD, Muchamuel T, Shen D, Wawrousek EF, et al. Inflammatory mediators in uveitis: differential induction of cytokines and chemokines in Th1- versus Th2- mediated ocular inflammation. J Immunol. 2002;168(5): 2483-92.

6. Huhtinen M, Karma A. HLA-B27 typing in the categorisation of uveitis in a HLA-B27 rich population. Br J Ophthalmol. 2000;84(4):413-6.

7. Konno Y, Numaga J, Tsuchiya N, Ogawa A, Islam SM, Mochizuki M, et al. HLA-B27 subtypes and HLA class II alleles in Japanese patients with anterior uveitis. Invest Ophthalmol Vis Sci. 1999;40(8):1838-44.

8. Martin TM, Kurz DE, Rosenbaum JT. Genetics of uveitis. Ophthalmol Clin North Am. 2003;16(4):555-65.

9. Niederer R, Danesh-Meyer H. Uveitis screening: HLAB27 antigen and ankylosing spondylitis in a New Zealand population. N Z Med J. 2006;119(1230):U1886.
10. Carvalho MAP, Campos WR, Araújo CAA, Lacerda RR, Oréfice F. Uveítes anteriores não granulomatosas, espondiloartropatias e HLA-B27. Rev Bras Reumatol. 1999;39(4):195-202

11. Gomariz EM, del M, Guijo VP, Contreras AE, Villanueva M, Estévez EC. The potential of ESSG spondyloarthropathy classification criteria as a diagnostic aid in rheumatological practice. J Rheumatol. 2002;29(2):326-30.

12. Sampaio-Barros PD, Bertolo MB, Kraemer MH, Neto JF, Samara AM Primary ankylosing spondylitis: patterns of disease in a Brazilian population of 147 patients. J Rheumatol. 2001;28(3):560-5.

13. Miyamoto N, Mandai M, Suzuma I, Suzuma K, Kobayashi K, Honda Y. Estrogen protects against cellular infiltration by reducing the expressions of Eselectin and IL-6 in endotoxin-induced uveitis. J Immunol. 1999;163(1):374-9.

14. Pato E, Bañares A, Jover JA, Fernández-Gutiérrez B, Gogoy F, Morado C, et al. Undiagnosed spondyloarthropathy in patients presenting with anterior uveitis. J Rheumatol. 2000;27(9):2198-202. 